\title{
ON MULTIPLE-PARTICLE CONTINUOUS-TIME RANDOM WALKS
}

\author{
PETER BECKER-KERN AND HANS-PETER SCHEFFLER
}

Received 18 August 2003 and in revised form 24 February 2004

Scaling limits of continuous-time random walks are used in physics to model anomalous diffusion in which particles spread at a different rate than the classical Brownian motion. In this paper, we characterize the scaling limit of the average of multiple particles, independently moving as a continuous-time random walk. The limit is taken by increasing the number of particles and scaling from microscopic to macroscopic view. We show that the limit is independent of the order of these limiting procedures and can also be taken simultaneously in both procedures. Whereas the scaling limit of a single-particle movement has quite an obscure behavior, the multiple-particle analogue has much nicer properties.

\section{Introduction}

Continuous-time random walks (CTRWs) were introduced in [24] to study random walks on a lattice. They are now used in physics to model a wide variety of phenomena connected with anomalous diffusion (see, e.g., [8, 9, 10, 13, 23, 29, 31, 34]). An approach different from CTRWs and fractional calculus to anomalous diffusion processes are the so-called random walks in random environments (see, e.g., [7, 12, 25] and the literature cited therein). However, this paper focuses on the CTRW approach, but it is an interesting open problem to discuss multiple-particle processes for random walks in random environments too. A CTRW is a random walk subordinated to a renewal process. The random walk increments represent the magnitude of particle jumps, and the renewal epochs represent the times of the particle jumps. CTRWs are also called renewal reward processes (see, e.g., [33] where applications are given to queuing theory). The usual assumption is that the CTRW is uncoupled, meaning that the random walk is independent of the subordinating renewal process. In this case, if the time between renewals has finite mean, then the renewal process is asymptotically equivalent to a constant multiple of the time variable, and the CTRW behaves like the original random walk for large time $[2,15]$. In many physical applications, the waiting time between renewals has infinite mean [30]. In [21], we showed that the scaling limit of an uncoupled CTRW with infinite mean waiting 
time is of the form $A(E(t))$, where $A(t)$ is the scaling limit of the underlying random walk and $E(t)$ is the hitting time process for a $\beta$-stable subordinator independent of $A(t)$.

In this paper, we analyze the limiting behavior of the average over multiple infinite mean waiting time CTRWs in the context of operator self-similarity of stochastic processes. As shown in [21], the limiting process $\{M(t)\}_{t \geq 0}$ of a single uncoupled CTRW has quite an obscure behavior, as it is not an operator-stable process nor it has independent increments (i.i.). It follows from [21, Theorem 4.6] that even for a Brownian motion $\{A(t)\}_{t \geq 0}$, the distribution of $M(t)$ is not even Gaussian.

We consider the average

$$
Z_{n}(t)=\frac{1}{n} \sum_{k=1}^{n} X^{(k)}(t)
$$

of independently moving particles, each moving as a CTRW $X^{(k)}(t)=S^{(k)}\left(N_{t}^{(k)}\right)$, where $S^{(k)}(n)$ denotes the random walk and $N_{t}^{(k)}$ denotes the renewal process. Now there appear three possible ways of central limiting behavior:

(1) first scale the model from microscopic to macroscopic view and then increase the number of particles with $n \rightarrow \infty$;

(2) first increase the number of particles with $n \rightarrow \infty$ and then scale the model from microscopic to macroscopic view;

(3) simultaneously increase the number of particles and scale the model, that is, analyze $Z_{n(c)}(c t)$, where $n(c) \rightarrow \infty$ as $c \rightarrow \infty$.

It will turn out that in any case of (1), (2), and (3), we get the same limiting process $\{\tilde{M}(t)\}_{t \geq 0}$ which is operator self-similar with exponent $F=\beta E$ and an operator-stable process in the sense of Maejima [17] with exponent $E$ and with independent but nonstationary increments.

Since we are interested in operator self-similarity, the appropriate mode of convergence is convergence in distribution of all finite-dimensional marginal distributions, denoted by $\stackrel{f . d}{\Rightarrow}$. The fact that the limiting behavior of $\left\{Z_{n}(t)\right\}_{t \geq 0}$ is the same in (1), (2), and (3) strongly suggests that the limiting process $\{\tilde{M}(t)\}_{t \geq 0}$ is a very robust model for anomalous diffusions with much nicer properties than the single-particle CTRW limit considered in $[14,21]$. In [14], the one-dimensional CTRW limit is considered and, by abuse of language, is called a fractional-stable distribution.

This paper is organized as follows. In Section 2, we define in detail a multiple-particle CTRW and state our basic assumptions necessary for our main results as well as some basic facts on operator-stable processes. In Section 3, our main results are presented together with its proofs. In Section 4, we compare the models for anomalous diffusions emerging from the one-particle versus the multiple-particle CTRW limiting processes. We conclude this paper by discussing an example of a so-called coupled CTRW, that is, a CTRW where the waiting times and the jumps are dependent. 


\section{Basic assumptions and preliminary results}

The formulation as well as the proofs of our results rely heavily on the multivariable central limit theory laid out in detail in [22]. In the following, we use the notation as well as some of the results of [22] without further citation. See also $[5,21]$ for more detailed references.

(A) The model. For a linear operator $Q$ on $\mathbb{R}^{d}$, let $\exp (Q)=\sum_{k=0}^{\infty}(k !)^{-1} Q^{k}$ denote the exponential and for $t>0$, define $t^{Q}=\exp (Q \log t)$. Let $Y, Y_{i}, Y_{i}^{(k)}, i, k \geq 1$, be i.i.d. $\mathbb{R}^{d_{-}}$ valued random vectors which model the particle jumps. For $t \geq 0$, let

$$
S(t)=\sum_{j=1}^{[t]} Y_{j}, \quad S^{(k)}(t)=\sum_{j=1}^{[t]} Y_{j}^{(k)}
$$

so that $S(t)=S^{(k)}(t)=0$ for $0 \leq t<1$ and assume that $Y$ belongs to the strict generalized domain of attraction of a full operator-stable random vector $A$ with exponent $E \in \mathrm{GL}\left(\mathbb{R}^{d}\right)$, where full means that $A$ is not concentrated on any proper hyperplane of $\mathbb{R}^{d}$. In summary, there exists a regularly varying norming function $B: \mathbb{R}_{+} \rightarrow \mathrm{GL}\left(\mathbb{R}^{d}\right)$ with exponent $-E$ (denoted by $B \in \mathrm{RV}(-E)$ ), that is, $B(\lambda t) B(t)^{-1} \rightarrow \lambda^{-E}$ as $t \rightarrow \infty$ for any $\lambda>0$, such that for any $t>0$,

$$
B(n) S(n t) \Longrightarrow A(t) \text { as } n \longrightarrow \infty
$$

where $A(t) \stackrel{d}{=} t^{E} A$ is the Lévy process generated by the operator-stable random vector $A$, which is called an operator Lévy motion. Here $\Rightarrow$ denotes convergence in distribution and $\stackrel{d}{=}$ denotes equality in distribution. Note that we have $\operatorname{Re}(\lambda) \geq 1 / 2$ for any eigenvalue $\lambda$ of the exponent $E$. Moreover, by independence (see [22, Example 11.2.18]), we obtain

$$
B(n) S^{(k)}(n t) \stackrel{\text { f.d. }}{\Longrightarrow} A^{(k)}(t) \text { as } n \longrightarrow \infty,
$$

where $\{A(t)\}_{t \geq 0},\left\{A^{(k)}(t)\right\}_{t \geq 0}, k \geq 1$, are i.i.d. operator-Lévy motions. Especially, [22, Example 11.2.18] shows that $\{A(t)\}_{t \geq 0}$ is operator self-similar with exponent $E$, that is, $\{A(c t)\}_{t \geq 0} \stackrel{f . d .}{=}\left\{c^{E} A(t)\right\}_{t \geq 0}$ for any $c>0$, where $\stackrel{f . d .}{=}$ denotes equality of all finite-dimensional marginal distributions.

Further, let $J, J_{i}, J_{i}^{(k)}$ be i.i.d. random variables with $J \geq 0$ almost surely that model the waiting times between successive jumps of the particles. For $t \geq 0$, let

$$
T(s)=\sum_{j=1}^{[s]} J_{j}, \quad T^{(k)}(s)=\sum_{j=1}^{[s]} J_{j}^{(k)}
$$

where again $T(t)=T^{(k)}(t)=0$ if $0 \leq t<1$ and assume that $J$ belongs to the domain of attraction of some $\beta$-stable random variable $D$ with $0<\beta<1$. To summarize this, there exists a regularly varying norming function $b: \mathbb{R}_{+} \rightarrow \mathbb{R}_{+}$with index $-1 / \beta$, that is, 
$b(\lambda t) b(t)^{-1} \rightarrow \lambda^{-1 / \beta}$ as $t \rightarrow \infty$ for any $\lambda>0$, such that for any $s>0$,

$$
b(n) T(n s) \Longrightarrow D(s) \text { as } n \longrightarrow \infty
$$

where $D(s) \stackrel{d}{=} s^{1 / \beta} D$ is a $\beta$-stable subordinator. Note that due to $0<\beta<1$, the random variable $J$ necessarily has infinite mean. Moreover, by independence we obtain

$$
b(n) T^{(k)}(n s) \stackrel{\text { f.d. }}{\Longrightarrow} D^{(k)}(s) \text { as } n \longrightarrow \infty,
$$

where $\{D(s)\}_{s \geq 0},\left\{D^{(k)}(s)\right\}_{t \geq 0}, k \geq 1$, are i.i.d. $\beta$-stable subordinators.

Now for $k \geq 1$ and $t \geq 0$, let $N_{t}^{(k)}=\max \left\{n \geq 0: T^{(k)}(n) \leq t\right\}$ denote the renewal process of the cumulative waiting times and let $E^{(k)}(t)=\inf \left\{s \geq 0: D^{(k)}(s)>t\right\}$ be the corresponding hitting time process by (2.6). Let $N_{t}$ be i.i.d. as $N_{t}^{(k)}, k \geq 1$, and let $E(t)$ be i.i.d. as $E^{(k)}(t), k \geq 1$. Note that $\{E(t)\}_{t \geq 0}$ has nondecreasing sampling paths and by [21, Corollary 3.2] has moments of all orders. Moreover, for some regularly varying function $\tilde{b}$ with index $\beta$ and $c \cdot b(\tilde{b}(c)) \rightarrow 1$ as $c \rightarrow \infty$, we have by [21, Theorem 3.6] that

$$
\frac{1}{\tilde{b}(c)} N_{c t}^{(k)} \stackrel{f \cdot d .}{\Longrightarrow} E^{(k)}(t) \quad \text { as } c \longrightarrow \infty
$$

for any $k \geq 1$.

Now for $k \geq 1$ and $t \geq 0$, let

$$
X^{(k)}(t)=S^{(k)}\left(N_{t}^{(k)}\right), \quad X(t)=S\left(N_{t}\right)
$$

be CTRWs, each describing the movement of a single-particle. Then $\left\{X^{(k)}(t)\right\}_{t \geq 0}$ are i.i.d. as $\{X(t)\}_{t \geq 0}$. In the following, we assume that $\left\{Y, Y_{i}, Y_{i}^{(k)}, J, J_{i}, J_{i}^{(k)}: i, k \geq 1\right\}$ are independent so that each CTRW $\left\{X^{(k)}(t)\right\}_{t \geq 0}$ is uncoupled. Let $\tilde{B}(c)=B(\tilde{b}(c))$. It follows from [21, Theorem 4.2] that for any $k \geq 1$,

$$
\tilde{B}(c) X^{(k)}(c t) \stackrel{f . d .}{\Longrightarrow} M^{(k)}(t)=A^{(k)}\left(E^{(k)}(t)\right) \quad \text { as } c \longrightarrow \infty,
$$

where $\left\{M^{(k)}(t)\right\}_{t \geq 0}$ are i.i.d. as $\{M(t)\}_{t \geq 0}=\{A(E(t))\}_{t \geq 0}$, each describing the macroscopic movement of a particle. It is shown in [21, Section 4] that $\left\{M^{(k)}(t)\right\}_{t \geq 0}$ is operator self-similar with exponent $F=\beta E$, that is,

$$
\left\{M^{(k)}(c t)\right\}_{t \geq 0} \stackrel{f . d .}{=}\left\{c^{F} M^{(k)}(t)\right\}_{t \geq 0}
$$

for any $k \geq 1$ and any $c>0$, but neither is operator-stable nor has stationary or independent increments.

(B) Operator-stable processes. In this section, we briefly recall the definition of an operator-stable process and analyze in detail the example of an operator Lévy motion, which is crucial for our main results. We follow the basic definition of [17]. Assume that $A$ is some strictly operator-stable random vector with exponent $E$ and distribution $\nu$, that is, $v$ is infinitely divisible and $v^{t}=\left(t^{E} v\right)$ for any $t>0$, where $v^{t}$ denotes the $t$-fold 
convolution power of $\nu$. It follows from [22, Theorem 7.2.1] that the real parts of the eigenvalues of $E$ are greater than or equal to $1 / 2$.

Definition 2.1. An $\mathbb{R}^{d}$-valued stochastic process $\{Z(t)\}_{t \geq 0}$ is called an operator-stable process with exponent $E$, if for any $0<t_{1}<\cdots<t_{m}$ and $m \geq 1$, the random vector $\left(Z\left(t_{1}\right), \ldots\right.$, $\left.Z\left(t_{m}\right)\right)$ is operator-stable with exponent $E_{(m)}=\operatorname{diag}(E, \ldots, E)$ on $\left(\mathbb{R}^{d}\right)^{m}$.

This definition generalizes the well-known notion of a Gaussian or symmetric $\alpha$-stable process. See [27] for details on those processes.

The following example is crucial for our main results: let $\{A(t)\}_{t \geq 0}$ be an operator Lévy motion with exponent $E$ and without normal component, that is, the distribution $v$ of $A=A(1)$ has the Lévy representation $[a, 0, \varphi]$, where $a \in \mathbb{R}^{d}$ and $\varphi$ denotes the Lévy measure of $\nu$ (see [22, Theorem 3.1.11]). It then follows from the operator stability of $\nu$ that $A(t)$ has distribution $\nu^{t}$ and hence by [22, Definition 3.1.23] has Lévy measure $t \cdot \varphi$. Then by [17], we have that $\{A(t)\}_{t \geq 0}$ is an operator-stable process in the sense of Definition 2.1. We now describe the Lévy measure of its finite-dimensional marginal distributions. Let $0=t_{0}<t_{1}<\cdots<t_{m}$ be arbitrary. Then the random vectors $A\left(t_{i}\right)-A\left(t_{i-1}\right), i=1, \ldots, m$, are independent and $A\left(t_{i}\right)-A\left(t_{i-1}\right) \stackrel{d}{=} A\left(t_{i}-t_{i-1}\right)$ has Lévy measure $\left(t_{i}-t_{i-1}\right) \cdot \varphi$. It follows from the independence of the $\mathbb{R}^{d}$-valued components that the $\left(\mathbb{R}^{d}\right)^{m}$-valued random vector

$$
\xi_{m}=\left(A\left(t_{1}\right), A\left(t_{2}\right)-A\left(t_{1}\right), \ldots, A\left(t_{m}\right)-A\left(t_{m-1}\right)\right)
$$

is operator-stable on $\left(\mathbb{R}^{d}\right)^{m}$ with exponent $E_{(m)}$ and has Lévy measure

$$
\tilde{\Phi}_{t_{1}, \ldots, t_{m}}=\sum_{i=1}^{m}\left(t_{i}-t_{i-1}\right) \cdot \varphi_{i}
$$

with

$$
\varphi_{i}=\varepsilon_{0} \otimes \cdots \otimes \varepsilon_{0} \otimes \varphi \otimes \varepsilon_{0} \otimes \cdots \otimes \varepsilon_{0}
$$

for $i=1, \ldots, m$, where $\varphi$ appears in the $i$ th component of the product measure and $\varepsilon_{0}$ denotes Dirac measure at the origin. Now let

$$
\Psi_{m}:\left(\mathbb{R}^{d}\right)^{m} \longrightarrow\left(\mathbb{R}^{d}\right)^{m}, \quad \Psi_{m}\left(x_{1}, \ldots, x_{m}\right)=\left(x_{1}, x_{1}+x_{2}, \ldots, x_{1}+\cdots+x_{m}\right)
$$

Then $\Psi_{m}$ is linear and invertible and we have $\Psi_{m} \circ t^{E_{(m)}}=t^{E_{(m)}} \circ \Psi_{m}$. Hence $\left(A\left(t_{1}\right), A\left(t_{2}\right)\right.$, $\left.\ldots, A\left(t_{m}\right)\right)=\Psi_{m}\left(\xi_{m}\right)$ is operator-stable on $\left(\mathbb{R}^{d}\right)^{m}$ with exponent $E_{(m)}$ and has Lévy measure

$$
\Phi_{t_{1}, \ldots, t_{m}}=\Psi_{m}\left(\tilde{\Phi}_{t_{1}, \ldots, t_{m}}\right)=\sum_{i=1}^{m}\left(t_{i}-t_{i-1}\right) \cdot \Psi_{m}\left(\varphi_{i}\right)
$$




\section{Main results}

In this section, we present our main results together with their proofs. We show that the multiple-particle average CTRW process $\left\{Z_{n}(t)\right\}_{t \geq 0}$ defined in (1.1) will, properly normalized, converge in the $\stackrel{f . d \text {. }}{\Longrightarrow}$-sense in all three cases (1), (2), and (3) to the same limiting process $\{\tilde{M}(t)\}_{t \geq 0}$. It will turn out that this limiting process is an operator-stable process with exponent $E$ which is operator self-similar with exponent $F=\beta E$, where $\beta$ and $E$ are as in Section 2. Let $f d-\mathscr{L}$ denote the convergence in distribution of finitedimensional marginals of the process.

Theorem 3.1 (case (1)).

$$
f d-\mathscr{L}-\lim _{n \rightarrow \infty} \lim _{c \rightarrow \infty} B(\tilde{b}(c) n) n Z_{n}(c t)=\tilde{M}(t)
$$

where $\{\tilde{M}(t)\}_{t \geq 0}$ is operator self-similar with exponent $F=\beta E$. Moreover, $\{\tilde{M}(t)\}_{t \geq 0}$ is an operator-stable process with exponent $E$. In case $A=A(1)$ in (2.3) has no normal component, for $0<t_{1}<\cdots<t_{m}$, the random vector $\left(\tilde{M}\left(t_{1}\right), \ldots, \tilde{M}\left(t_{m}\right)\right)$ has Lévy measure

$$
\Xi_{t_{1}, \ldots, t_{m}}=\Phi_{C_{\beta} t_{1}^{\beta}, \ldots, C_{\beta} t_{m}^{\beta}},
$$

where $\Phi_{s_{1}, \ldots, s_{m}}$ is as in (2.15) and $C_{\beta}=\mathbb{E}(E(1)) ; \mathbb{E}$ denoting expectation. Especially, $\{\tilde{M}(t)\}_{t \geq 0}$ has independent increments and $\tilde{M}(t)-\tilde{M}(s) \stackrel{d}{=}\left(C_{\beta}\left(t^{\beta}-s^{\beta}\right)\right)^{E} A$ for $0 \leq s<t$.

Theorem 3.2 (case (2)).

$$
f d-\mathscr{L}-\lim _{c \rightarrow \infty} \lim _{n \rightarrow \infty} B(\tilde{b}(c) n) n Z_{n}(c t)=\tilde{M}(t)
$$

where the limiting process $\{\tilde{M}(t)\}_{t \geq 0}$ is as in Theorem 3.1.

Theorem 3.3 (case (3)). Let $n(c) \rightarrow \infty$ as $c \rightarrow \infty$. Then

$$
f d-\mathscr{L}-\lim _{c \rightarrow \infty} B(\tilde{b}(c) n(c)) n(c) Z_{n(c)}(c t)=\tilde{M}(t)
$$

where the limiting process $\{\tilde{M}(t)\}_{t \geq 0}$ is as in Theorem 3.1.

We now give the proofs of our main results together with a technical lemma necessary for the proofs. We start with Theorem 3.1.

Proof of Theorem 3.1. Fix any $0=t_{0}<t_{1}<\cdots<t_{m}$. Let for all $i$ mean for $i=1, \ldots, m$. It follows from [21, Theorem 4.2] (see (2.9)) that for all $k \geq 1$, we have

$$
\left(\tilde{B}(c) X^{(k)}\left(c t_{i}\right): \forall i\right) \Longrightarrow\left(M^{(k)}\left(t_{i}\right): \forall i\right) \quad \text { as } c \longrightarrow \infty
$$


Since $B \in \mathrm{RV}(-E)$, we know $B(\tilde{b}(c) n) B(\tilde{b}(c))^{-1} \rightarrow n^{-E}$ as $c \rightarrow \infty$. Hence, by independence in $k$, we conclude that

$$
\begin{aligned}
\left(B(\tilde{b}(c) n) n Z_{n}\left(c t_{i}\right): \forall i\right) & =\sum_{k=1}^{n}\left(\left(B(\tilde{b}(c) n) B(\tilde{b}(c))^{-1}\right) \tilde{B}(c) X^{(k)}\left(c t_{i}\right): \forall i\right) \\
& \Longrightarrow \sum_{k=1}^{n}\left(n^{-E} M^{(k)}\left(t_{i}\right): \forall i\right) \quad \text { as } c \longrightarrow \infty .
\end{aligned}
$$

Now for any Borel sets $U_{i} \subset \mathbb{R}^{d}, i=1, \ldots, m$, we have by independence of $\left\{A^{(k)}(t)\right\}_{t \geq 0}$ and $\left\{E^{(k)}(t)\right\}_{t \geq 0}$ for any $k$ that

$$
\begin{aligned}
& P\left\{\sum_{k=1}^{n}\left(M^{(k)}\left(t_{i}\right): \forall i\right) \in U_{1} \times \cdots \times U_{m}\right\} \\
& =P\left\{\sum_{k=1}^{n} A^{(k)}\left(E^{(k)}\left(t_{1}\right)\right) \in U_{1}, \ldots, \sum_{k=1}^{n} A^{(k)}\left(E^{(k)}\left(t_{m}\right)\right) \in U_{m}\right\} \\
& =\int_{\mathbb{R}_{+}^{m}} \cdots \int_{\mathbb{R}_{+}^{m}} P\left\{\sum_{k=1}^{n} A^{(k)}\left(x_{k}^{(1)}\right) \in U_{1}, \ldots, \sum_{k=1}^{n} A^{(k)}\left(x_{k}^{(m)}\right) \in U_{m}\right\} \\
& =\int_{\mathbb{R}_{+}^{m}} \cdots \int_{\mathbb{R}_{+}^{m}} P\left\{A\left(x_{1}^{(1)}+\cdots+x_{n}^{(1)}\right) \in U_{1}, \ldots, A\left(x_{1}^{(m)}+\cdots+x_{n}^{(m)}\right) \in U_{m}\right\} \\
& =P\left\{A\left(\sum_{k=1}^{n} E^{(k)}\left(t_{1}\right)\right) \in U_{1}, \ldots, A\left(\sum_{k=1}^{n} E^{(k)}\left(t_{m}\right)\right) \in U_{m}\right\} .
\end{aligned}
$$

In order to justify the formula above, we have to show that

$$
\begin{aligned}
& P\left\{\sum_{k=1}^{n} A^{(k)}\left(x_{k}^{(1)}\right) \in U_{1}, \ldots, \sum_{k=1}^{n} A^{(k)}\left(x_{k}^{(m)}\right) \in U_{m}\right\} \\
& \quad=P\left\{A\left(x_{1}^{(1)}+\cdots+x_{n}^{(1)}\right) \in U_{1}, \ldots, A\left(x_{1}^{(m)}+\cdots+x_{n}^{(m)}\right) \in U_{m}\right\} .
\end{aligned}
$$

For $m=1$, we have by independence that

$$
P\left\{\sum_{k=1}^{n} A^{(k)}\left(x_{k}^{(1)}\right) \in U_{1}\right\}=P\left\{A\left(x_{1}^{(1)}+\cdots+x_{n}^{(1)}\right) \in U_{1}\right\} .
$$

Note that since the sample paths of $\{E(t)\}_{t \geq 0}$ are nondecreasing, we necessarily have $x_{i}^{(1)} \leq \cdots \leq x_{i}^{(m)}$ for all $i=1, \ldots, n$. By induction and using the fact that $\left\{A^{(k)}(t)\right\}_{t \geq 0}$ has 
stationary and independent increments, we therefore get that

$$
\begin{aligned}
& P\left\{\sum_{k=1}^{n} A^{(k)}\left(x_{k}^{(1)}\right) \in U_{1}, \ldots, \sum_{k=1}^{n} A^{(k)}\left(x_{k}^{(m)}\right) \in U_{m}\right\} \\
& =P\left\{\sum_{k=1}^{n} A^{(k)}\left(x_{k}^{(1)}\right) \in U_{1}, \ldots, \sum_{k=1}^{n} A^{(k)}\left(x_{k}^{(m-1)}\right) \in U_{m-1},\right. \\
& \left.\sum_{k=1}^{n}\left(A^{(k)}\left(x_{k}^{(m-1)}\right)+\left(A^{(k)}\left(x_{k}^{(m)}\right)-A^{(k)}\left(x_{k}^{(m-1)}\right)\right)\right) \in U_{m}\right\} \\
& =\int_{\mathbb{R}^{d}} \cdots \int_{\mathbb{R}^{d}} P\left\{\sum_{k=1}^{n} A^{(k)}\left(x_{k}^{(1)}\right) \in U_{1}, \ldots, \sum_{k=1}^{n} A^{(k)}\left(x_{k}^{(m-1)}\right) \in U_{m-1},\right. \\
& \left.\sum_{k=1}^{n}\left(A^{(k)}\left(x_{k}^{(m-1)}\right)+y_{k}\right) \in U_{m}\right\} \\
& \times d P_{A\left(x_{1}^{(m)}-x_{1}^{(m-1)}\right)}\left(y_{1}\right) \cdots d P_{A\left(x_{n}^{(m)}-x_{n}^{(m-1)}\right)}\left(y_{n}\right) \\
& =\int_{\mathbb{R}^{d}} \cdots \int_{\mathbb{R}^{d}} P\left\{A\left(x_{1}^{(1)}+\cdots+x_{n}^{(1)}\right) \in U_{1}, \ldots, A\left(x_{1}^{(m-1)}+\cdots+x_{n}^{(m-1)}\right) \in U_{m-1},\right. \\
& \left.A\left(x_{1}^{(m-1)}+\cdots+x_{n}^{(m-1)}\right)+y_{1}+\cdots+y_{n} \in U_{m}\right\} \\
& \times d P_{A\left(x_{1}^{(m)}-x_{1}^{(m-1)}\right)}\left(y_{1}\right) \cdots d P_{A\left(x_{n}^{(m)}-x_{n}^{(m-1)}\right)}\left(y_{n}\right) \\
& =P\left\{A\left(x_{1}^{(1)}+\cdots+x_{n}^{(1)}\right) \in U_{1}, \ldots, A\left(x_{1}^{(m-1)}+\cdots+x_{n}^{(m-1)}\right) \in U_{m-1}\right. \text {, } \\
& \left.A\left(x_{1}^{(m-1)}+\cdots+x_{n}^{(m-1)}+\sum_{k=1}^{n}\left(x_{k}^{(m)}-x_{k}^{(m-1)}\right)\right) \in U_{m}\right\} \\
& =P\left\{A\left(x_{1}^{(1)}+\cdots+x_{n}^{(1)}\right) \in U_{1}, \ldots, A\left(x_{1}^{(m)}+\cdots+x_{n}^{(m)}\right) \in U_{m}\right\} \text {, }
\end{aligned}
$$

proving (3.8).

Therefore, by (3.6) and (3.7), we have shown that

$$
\left\{B(\tilde{b}(c) n) n Z_{n}(t)\right\}_{t \geq 0} \stackrel{\text { f.d. }}{\Longrightarrow}\left\{n^{-E} A\left(\sum_{k=1}^{n} E^{(k)}(t)\right)\right\}_{t \geq 0}
$$

as $c \rightarrow \infty$. Since by [21, Corollary 3.2], we have $\mathbb{E}\left(E(t)^{\gamma}\right)=C_{\beta} t^{\beta \gamma}$ for any $\gamma>0$, the weak law of large numbers implies

$$
\frac{1}{n} \sum_{k=1}^{n} E^{(k)}\left(t_{i}\right) \longrightarrow \mathbb{E}\left(E\left(t_{i}\right)\right) \quad \text { as } n \longrightarrow \infty
$$


in probability for $1 \leq i \leq m$. In view of (3.7), we therefore get for any continuity sets $U_{i} \subset \mathbb{R}^{d}, i=1, \ldots, m$, using that $\{A(c t)\}_{t \geq 0} \stackrel{f . d .}{=}\left\{c^{E} A(t)\right\}_{t \geq 0}$,

$$
\begin{aligned}
P\left\{\sum_{k=1}^{n}\right. & \left.\left(n^{-E} M^{(k)}\left(t_{i}\right): \forall i\right) \in U_{1} \times \cdots \times U_{m}\right\} \\
& =P\left\{n^{-E} A\left(\sum_{k=1}^{n} E^{(k)}\left(t_{1}\right)\right) \in U_{1}, \ldots, n^{-E} A\left(\sum_{k=1}^{n} E^{(k)}\left(t_{m}\right)\right) \in U_{m}\right\} \\
& =P\left\{A\left(\frac{1}{n} \sum_{k=1}^{n} E^{(k)}\left(t_{1}\right)\right) \in U_{1}, \ldots, A\left(\frac{1}{n} \sum_{k=1}^{n} E^{(k)}\left(t_{m}\right)\right) \in U_{m}\right\} \\
& \longrightarrow P\left\{A\left(\mathbb{E}\left(E\left(t_{1}\right)\right)\right) \in U_{1}, \ldots, A\left(\mathbb{E}\left(E\left(t_{m}\right)\right)\right) \in U_{m}\right\}
\end{aligned}
$$

as $n \rightarrow \infty$, using the continuity in distribution of $\left(y_{1}, \ldots, y_{m}\right) \mapsto\left(A\left(y_{1}\right), \ldots, A\left(y_{m}\right)\right)$ and (3.12). Hence (3.1) follows, where

$$
\{\tilde{M}(t)\}_{t \geq 0} \stackrel{f . d .}{=}\{A(\mathbb{E}(E(t)))\}_{t \geq 0}=\left\{A\left(C_{\beta} t^{\beta}\right)\right\}_{t \geq 0} .
$$

Hence $\{\tilde{M}(t)\}_{t \geq 0}$ has independent increments with $\tilde{M}(t)-\tilde{M}(s) \stackrel{d}{=}\left(C_{\beta}\left(t^{\beta}-s^{\beta}\right)\right)^{E} A$. Moreover,

$$
\left\{c^{F} \tilde{M}(t)\right\}_{t \geq 0} \stackrel{f . d .}{=}\left\{\left(c^{\beta}\right)^{E} A\left(C_{\beta} t^{\beta}\right)\right\}_{t \geq 0} \stackrel{\text { f.d. }}{=}\left\{A\left(C_{\beta} c^{\beta} t^{\beta}\right)\right\}_{t \geq 0} \stackrel{f . d .}{=}\{\tilde{M}(c t)\}_{t \geq 0},
$$

proving that $\{\tilde{M}(t)\}_{t \geq 0}$ is operator self-similar with exponent $F=\beta E$. Finally, if $A$ has no normal component and Lévy measure $\varphi$, by (2.15) the operator-stable random vector $\left(A\left(s_{1}\right), \ldots, A\left(s_{m}\right)\right)$ for $0<s_{1}<\cdots<s_{m}$ has Lévy measure $\Phi_{s_{1}, \ldots, s_{m}}$. Hence the Lévy measure of the operator-stable random vector $\left(\tilde{M}\left(t_{1}\right), \ldots, \tilde{M}\left(t_{m}\right)\right)$ with exponent $E_{(m)}$ has Lévy measure $\Xi_{t_{1}, \ldots, t_{m}}$ as in (3.2). This concludes the proof.

For the proof of Theorems 3.2 and 3.3, we need the following lemma, which might be of independent interest.

Lemma 3.4. For any $\gamma>0$, we have

$$
\mathbb{E}\left(\left(\frac{1}{\tilde{b}(c)} N_{c t}\right)^{\gamma}\right) \rightarrow \mathbb{E}\left(E(t)^{\gamma}\right) \quad \text { as } c \longrightarrow \infty .
$$

Proof. Note that

$$
\mathbb{E}\left(\left(\frac{1}{\tilde{b}(c)} N_{c t}\right)^{\gamma}\right)=\gamma \int_{0}^{\infty} x^{\gamma-1} P\left\{\frac{1}{\tilde{b}(c)} N_{c t} \geq x\right\} d x
$$

and that by [21, Theorem 3.6], we have

$$
P\left\{\frac{1}{\tilde{b}(c)} N_{c t} \geq x\right\} \rightarrow P\{E(t) \geq x\} \quad \text { as } c \longrightarrow \infty .
$$


We now show that for some small $\delta>0$, there exists a $c_{0}>0$ such that

$$
P\left\{\frac{1}{\tilde{b}(c)} N_{c t} \geq x\right\} \leq e^{t} e^{(-1+\delta) x}
$$

for all $c \geq c_{0}$ and all $x>0$. Then by dominated convergence, we obtain

$$
\gamma \int_{0}^{\infty} x^{\gamma-1} P\left\{\frac{1}{\tilde{b}(c)} N_{c t} \geq x\right\} \longrightarrow \gamma \int_{0}^{\infty} x^{\gamma-1} P\{E(t) \geq x\} d x=\mathbb{E}\left(E(t)^{\gamma}\right)
$$

as $c \rightarrow \infty$.

It remains to show (3.19). Since $b(\tilde{b}(c)) \sim 1 / c$ as $c \rightarrow \infty$, we have $c^{-1} T(\tilde{b}(c)) \Rightarrow D$ as $c \rightarrow \infty$. In view of [28, Example 24.12], for a suitable choice of the norming function $b$ in (2.6), we get for the Laplace transform $\mathbb{E}\left(e^{-s D}\right)=\exp \left(-s^{\beta}\right)$ for $s \geq 0$. Hence by the continuity theorem for Laplace transforms, we have for any $s \geq 0$ that

$$
\mathbb{E}\left(e^{-s c^{-1} T(\tilde{b}(c))}\right) \longrightarrow \mathbb{E}\left(e^{-s D}\right)=e^{-s^{\beta}} \quad \text { as } c \longrightarrow \infty .
$$

Therefore, for $s=1$, we obtain

$$
\left(\mathbb{E}\left(e^{-c^{-1} J}\right)\right)^{[\tilde{b}(c)]} \longrightarrow e^{-1} \text { as } c \longrightarrow \infty
$$

so for any $0<\delta<1$, there exists a $c_{0}>0$ such that

$$
\left(\mathbb{E}\left(e^{-c^{-1} J}\right)\right)^{\tilde{b}(c)} \leq e^{-1+\delta} \quad \forall c \geq c_{0} .
$$

Since $\{T(n) \leq t\}=\left\{N_{t} \geq n\right\}$, using Markov's inequality, we have

$$
\begin{aligned}
P\left\{\frac{1}{\tilde{b}(c)} N_{c t} \geq x\right\} & =P\left\{N_{c t} \geq \tilde{b}(c) x\right\} \\
& =P\left\{c^{-1} T(\tilde{b}(c) x) \leq t\right\} \\
& =P\left\{\exp \left(-c^{-1} T(\tilde{b}(c) x)\right) \geq e^{-t}\right\} \\
& \leq e^{t} \mathbb{E}\left(\exp \left(-c^{-1} T(\tilde{b}(c) x)\right)\right) \\
& =e^{t}\left(\mathbb{E}\left(e^{-c^{-1} J}\right)\right)^{\tilde{b}(c) x} \\
& \leq e^{t} e^{(-1+\delta) x}
\end{aligned}
$$

for all $c \geq c_{0}$ and all $x>0$, proving (3.19). This concludes the proof.

Proof of Theorem 3.2. Again, fix any $0<t_{1}<\cdots<t_{m}$ and note that by (2.3) and independence, we have for all $k \geq 1$ that

$$
\left(B(n) S^{(k)}\left(n t_{i}\right): \forall i\right) \Longrightarrow\left(A^{(k)}\left(t_{i}\right): \forall i\right) \quad \text { as } n \longrightarrow \infty
$$


Similar to the proof of (3.7) and (3.8), we have for any Borel sets $U_{i} \subset \mathbb{R}^{d}$ that

$$
\begin{aligned}
P\left\{\sum_{k=1}^{n}\left(X^{(k)}\left(t_{i}\right): \forall i\right) \in U_{1} \times \cdots \times U_{m}\right\} & \\
= & P\left\{\sum_{k=1}^{n} S^{(k)}\left(N_{t_{1}}^{(k)}\right) \in U_{1}, \ldots, \sum_{k=1}^{n} S^{(k)}\left(N_{t_{m}}^{(k)}\right) \in U_{m}\right\} \\
= & P\left\{S\left(\sum_{k=1}^{n} N_{t_{1}}^{(k)}\right) \in U_{1}, \ldots, S\left(\sum_{k=1}^{n} N_{t_{m}}^{(k)}\right) \in U_{m}\right\} .
\end{aligned}
$$

Using (3.25), we therefore get for any continuity sets $U_{i} \subset \mathbb{R}^{d}$ that

$$
\begin{gathered}
P\left\{\left(B(\tilde{b}(c) n) n Z_{n}\left(c t_{i}\right): \forall i\right) \in U_{1} \times \cdots \times U_{m}\right\} \\
=P\left\{B(\tilde{b}(c) n) S\left(\sum_{k=1}^{n} N_{c t_{1}}^{(k)}\right) \in U_{1}, \ldots, B(\tilde{b}(c) n) S\left(\sum_{k=1}^{n} N_{c t_{m}}^{(k)}\right) \in U_{m}\right\} \\
=\int_{\mathbb{R}_{+}^{m}} P\left\{B(\tilde{b}(c) n) S\left(n x_{1}\right) \in U_{1}, \ldots, B(\tilde{b}(c) n) S\left(n x_{m}\right) \in U_{m}\right\} \\
\quad \times d P_{\left((1 / n) \sum_{k=1}^{n} N_{c t_{1}}^{(k)}, \ldots,(1 / n) \sum_{k=1}^{n} N_{c t_{m}}^{(k)}\right)}\left(x_{1}, \ldots, x_{m}\right) .
\end{gathered}
$$

Note that by $[4,(4.3)]$, together with the regular variation of $B$, that is, $B(\tilde{b}(c) n) B(n)^{-1} \rightarrow$ $\tilde{b}(c)^{-E}$, we have

$$
P\left\{B(\tilde{b}(c) n) S\left(n x_{i}\right) \in U_{i}: \forall i\right\} \longrightarrow P\left\{\tilde{b}(c)^{-E} A\left(x_{i}\right) \in U_{i}: \forall i\right\}
$$

as $n \rightarrow \infty$ uniformly on compact subsets of $\left\{0 \leq x_{1} \leq \cdots \leq x_{m}\right\}$. Moreover, by the weak law of large numbers, we have $n^{-1} \sum_{k=1}^{n} N_{c t_{i}}^{(k)} \rightarrow \mathbb{E}\left(N_{c t_{i}}\right)$ as $n \rightarrow \infty$ in probability and hence in distribution. Then by [4, Proposition 4.1], we conclude

$$
P\left\{\left(B(\tilde{b}(c) n) n Z_{n}\left(c t_{i}\right): \forall i\right) \in U_{1} \times \cdots \times U_{m}\right\} \longrightarrow P\left\{\tilde{b}(c)^{-E} A\left(\mathbb{E}\left(N_{c t_{i}}\right)\right) \in U_{i}: \forall i\right\}
$$

as $n \rightarrow \infty$.

In view of the operator self-similarity of $\{A(t)\}_{t \geq 0}$ with exponent $E$ together with Lemma 3.4, we finally obtain

$$
\begin{aligned}
P\left\{\tilde{b}(c)^{-E} A\left(\mathbb{E}\left(N_{c t_{i}}\right)\right) \in U_{i}: \forall i\right\} & =P\left\{A\left(\mathbb{E}\left(\frac{1}{\tilde{b}(c)} N_{c t_{i}}\right)\right) \in U_{i}: \forall i\right\} \\
& \longrightarrow P\left\{A\left(\mathbb{E}\left(E\left(t_{i}\right)\right)\right) \in U_{i}: \forall i\right\}
\end{aligned}
$$

as $c \rightarrow \infty$ which concludes the proof. 
Proof of Theorem 3.3. We argue as before. Fix any $0<t_{1}<\cdots<t_{m}$ and continuity sets $U_{i} \in \mathbb{R}^{d}$. Using (3.26) and using the independence of $\left\{S^{(k)}(t)\right\}_{t \geq 0}$ and $\left\{N_{t}^{(k)}\right\}_{t \geq 0}$ again, we have that

$$
\begin{aligned}
P\{( & \left.\left.(\tilde{b}(c) n(c)) n(c) Z_{n(c)}\left(c t_{i}\right): \forall i\right) \in U_{1} \times \cdots \times U_{m}\right\} \\
\quad & P\left\{B(\tilde{b}(c) n(c)) \sum_{k=1}^{n(c)} S^{(k)}\left(N_{c t_{i}}^{(k)}\right) \in U_{i}: \forall i\right\} \\
& =P\left\{B(\tilde{b}(c) n(c)) S\left(\tilde{b}(c) n(c) \cdot \frac{1}{n(c)} \sum_{k=1}^{n(c)} \frac{1}{\tilde{b}(c)} N_{c t_{i}}^{(k)}\right) \in U_{i}: \forall i\right\} \\
& =\int_{\mathbb{R}_{+}^{m}} P\left\{B(\tilde{b}(c) n(c)) S\left(\tilde{b}(c) n(c) x_{i}\right) \in U_{i}: \forall i\right\} d P_{\left(T_{c}^{(1)}, \ldots, T_{c}^{(m)}\right)}\left(x_{1}, \ldots, x_{m}\right),
\end{aligned}
$$

where for $i=1, \ldots, m$,

$$
T_{c}^{(i)}=\frac{1}{n(c)} \sum_{k=1}^{n(c)} \frac{1}{\tilde{b}(c)} N_{c t_{i}}^{(k)}
$$

Now by Lemma 3.4, we have $\mathbb{E}\left(\tilde{b}(c)^{-1} N_{c t_{i}}\right) \rightarrow \mathbb{E}\left(E\left(t_{i}\right)\right)$ as $c \rightarrow \infty$ and by Tschebyschev's inequality,

$$
\begin{aligned}
P\left\{\left|T_{c}^{(i)}-\mathbb{E}\left(\frac{1}{\tilde{b}(c)} N_{c t_{i}}\right)\right|>\varepsilon\right\} & =P\left\{\left|\frac{1}{n(c)} \sum_{k=1}^{n(c)} \frac{1}{\tilde{b}(c)}\left(N_{c t_{i}}^{(k)}-\mathbb{E}\left(N_{c t_{i}}\right)\right)\right|>\varepsilon\right\} \\
& \leq \frac{1}{\varepsilon^{2}} \mathbb{V}\left(\frac{1}{n(c)} \sum_{k=1}^{n(c)} \frac{1}{\tilde{b}(c)}\left(N_{c t_{i}}^{(k)}-\mathbb{E}\left(N_{c t_{i}}\right)\right)\right) \\
& =\frac{1}{\varepsilon^{2}} \frac{1}{n(c)} \mathbb{V}\left(\frac{1}{\tilde{b}(c)} N_{c t_{i}}\right) \rightarrow 0
\end{aligned}
$$

as $c \rightarrow \infty$, using Lemma 3.4 again. Hence $T_{c}^{(i)} \rightarrow \mathbb{E}\left(E\left(t_{i}\right)\right)$ in probability as $c \rightarrow \infty$ for any $i=1, \ldots, m$ and in view of (3.25) and (3.31), we get by the same argument as in the proof of Theorem 3.2 that

$$
P\left\{B(\tilde{b}(c) n(c)) n(c) Z_{n(c)}\left(c t_{i}\right) \in U_{i}: \forall i\right\} \longrightarrow P\left\{A\left(\mathbb{E}\left(E\left(t_{i}\right)\right)\right) \in U_{i}: \forall i\right\}
$$

as $c \rightarrow \infty$ and the proof is complete.

Remark 3.5. It is shown in [16, Section 5] that in case of a Lévy motion $\{A(t)\}_{t \geq 0}$ without normal component, the distribution of the scaling limit $M(t)=A(E(t))$ of a single CTRW is regularly varying with exponent $E$ in the sense of [22, Definition 6.1.1] and hence belongs to the generalized domain of attraction of $A=A(1)$. The proof of Theorem 3.2 shows that the distribution of $X(t)$ for any fixed $t>0$ belongs to the generalized domain 
of attraction of $A$ as well and hence, by [22, Corollary 8.2.11], also has a regularly varying distribution with exponent $E$.

Remark 3.6. Note that since $t \mapsto \mathbb{E}(E(t))=C_{\beta} t^{\beta}$ is continuous and $\{A(t)\}_{t \geq 0}$ is a Lévy process, the Hausdorff dimension of the range of the sample paths of the limiting process is

$$
\operatorname{dim} \tilde{M}([0,1])=\operatorname{dim} A(\mathbb{E}(E[0,1]))=\operatorname{dim} A([0,1]) \quad \text { almost surely. }
$$

Now $\operatorname{dim} A([0,1])$ can be computed using the formulas in [3, Theorems 2.1 and 2.2] which only depend on the real parts of the eigenvalues of the exponent $E$ together with the dimensions of the corresponding generalized eigenspaces. Hence the operator stability exponent $E$ of $\{\tilde{M}(t)\}_{t \geq 0}$ determines the tail behavior and thus the Hausdorff dimension of the sample paths, whereas the operator self-similarity exponent $F=\beta E$ determines the space-time scaling behavior.

Remark 3.7. We now present the governing equation for the density $q(x, t)$ of the distribution of $\tilde{M}(t)$, where $\{\tilde{M}(t)\}_{t \geq 0}$ is the limiting process obtained in Section 3. Recall from [11, Section 2] that the density $p(x, t)$ of $A(t)$, where $\{A(t)\}_{t \geq 0}$ is an operator Lévy motion, solves the pseudodifferential equation

$$
\frac{\partial p}{\partial t}(x, t)=L_{x} p(x, t), \quad p(x, 0)=\delta(x)
$$

where $L_{x}$ is the generator of the corresponding continuous convolution semigroup of probability measures of $\{A(t)\}_{t \geq 0}$. The suffix $x$ indicates that $L_{x}$ is only acting on the space variable $x$. Note that $L_{x}$ is a (nonlocal) pseudodifferential operator generated by the logcharacteristic function of the distribution of $A(1)$. See $[19,20]$ for further information and applications to hydrology. If $\{A(t)\}_{t \geq 0}$ is a standard Brownian motion, then $L_{x}=$ $\Delta_{x}=\sum_{i=1}^{d}\left(\partial^{2} / \partial x_{i}^{2}\right)$ is the Laplace operator.

Recall from Section 3 that $\tilde{M}(t)=A\left(C_{\beta} t^{\beta}\right)$ and hence if $q(x, t)$ denotes the density of $\tilde{M}(t)$, we have $q(x, t)=p\left(x, C_{\beta} t^{\beta}\right)$. Since $\tilde{M}(0)=A(0)=0$ almost surely, it follows from (3.36) that $q(x, t)$ solves the pseudodifferential equation

$$
\frac{\partial}{\partial t} q(x, t)=L_{x}(t) q(x, t), \quad q(x, 0)=\delta(x)
$$

where $L_{x}(t)=C_{\beta} \beta t^{\beta-1} L_{x}$ and $L_{x}$ is the generator of $\{A(t)\}_{t \geq 0}$. Here $\delta(x)$ denotes the Dirac distribution. Note that since $0<\beta<1$, the now time-dependent generator $L_{x}(t)$ has a singularity at $t=0$.

Now, if the real parts of the eigenvalues of $E$ are smaller than $1 / \beta$, it follows from [18, Theorem 5.4] that the fractional operator Lévy motion

$$
\Theta(t)=\Theta_{\beta E, E}(t)=\int_{-\infty}^{\infty}\left(|t-u|^{(\beta-1) E}-|u|^{(\beta-1) E}\right) d Z(u)
$$


where $\{Z(t)\}_{t \in \mathbb{R}}$ is an operator Lévy motion with exponent $E$, exists and $\{\Theta(t)\}_{t \geq 0}$ is operator self-similar with exponent $F=\beta E$ and an operator-stable process with exponent $E$, similar to our limiting process $\{\tilde{M}(t)\}_{t \geq 0}$. Now, using the operator self-similarity and operator stability, we obtain

$$
\Theta(t) \stackrel{d}{=} t^{F} \Theta(1) \stackrel{d}{=}\left(t^{\beta}\right)^{E} \Theta(1) \stackrel{d}{=} \tilde{A}\left(t^{\beta}\right)
$$

for some operator Lévy motion $\{\tilde{A}(t)\}_{t \geq 0}$ with exponent $E$, generated by $\tilde{A}(1)=\Theta(1)$. Hence, the density $f(x, t)$ of $\Theta(t)$ also solves a variant of (3.37), with $L_{x}$ replaced by the generator of $\{\tilde{A}(t)\}_{t \geq 0}$. Therefore, we have $\Theta(t) \stackrel{d}{=} \tilde{A}\left(t^{\beta}\right)$ for any $t>0$. The difference between the two processes $\{\Theta(t)\}_{t \geq 0}$ and $\left\{\tilde{A}\left(t^{\beta}\right)\right\}_{t \geq 0}$ is that $\left\{\tilde{A}\left(t^{\beta}\right)\right\}_{t \geq 0}$ has independent but nonstationary increments, whereas $\{\Theta(t)\}_{t \geq 0}$ has stationary but no independent increments.

\section{Comparison of anomalous diffusion models}

In this section, we compare the two different models for anomalous diffusions emerging from the single-particle CTRW scaling limit and the multiple-particle CTRW limiting process. Our discussion includes stochastic properties of the limiting process as well as the corresponding pseudodifferential equations for their densities.

We start with the single-particle CTRW scaling limit $\{M(t)\}_{t \geq 0}$. Complementary to the results in [21], we first provide the following result which is of independent interest. Recall from [21, Theorem 4.2] that $M(t)=A(E(t))$, where $\{A(t)\}_{t \geq 0}$ is the operator Lévy motion modelling the jumps and $\{E(t)\}_{t \geq 0}$ is the hitting time process of a $\beta$-stable subordinator. Note that both processes are independent.

Theorem 4.1. Let $\{M(t)\}_{t \geq 0}$ be the scaling limit of an uncoupled CTRW. Then $\{M(t)\}_{t \geq 0}$ does not have independent increments.

Proof. Let $v$ denote the distribution of $A(1)$. Then the strict operator stability implies that $\nu^{t}=t^{E} \nu$ for all $t>0$. Hence, if $\hat{\nu}(\xi)=\exp (\psi(\xi))$ with $\log$-characteristic function $\psi$, we obtain

$$
t \cdot \psi(\xi)=\psi\left(t^{E^{*}} \xi\right) \quad \forall t>0, \xi \in \mathbb{R}^{d} .
$$

Assume that $\{M(t)\}_{t \geq 0}$ has independent increments. Fix any $0<t_{1}<t_{2}$. Then, using the i.i. assumption, we get that for any $\xi_{1}, \xi_{2} \in \mathbb{R}^{d}$,

$$
\begin{aligned}
& \mathbb{E}\left[\exp \left(i\left\langle\xi_{1}, M\left(t_{2}\right)-M\left(t_{1}\right)\right\rangle+i\left\langle\xi_{2}, M\left(t_{1}\right)\right\rangle\right)\right] \\
& \quad=\mathbb{E}\left[\exp \left(i\left\langle\xi_{1}, M\left(t_{2}\right)-M\left(t_{1}\right)\right\rangle\right)\right] \cdot \mathbb{E}\left[\exp \left(i\left\langle\xi_{2}, M\left(t_{1}\right)\right\rangle\right)\right] \\
& \quad=\tilde{R}\left(t_{1}, t_{2} ; \xi_{1}, \xi_{2}\right) .
\end{aligned}
$$


Now, since $\{A(t)\}_{t \geq 0}$ has stationary and independent increments (s.i.i.), we obtain

$$
\begin{aligned}
\mathbb{E}\left[\exp \left(i\left\langle\xi_{1}, M\left(t_{2}\right)-M\left(t_{1}\right)\right\rangle\right)\right] & =\int \mathbb{E}\left[\exp \left(i\left\langle\xi_{1}, A(v)-A(u)\right\rangle\right)\right] d P_{\left(E\left(t_{1}\right), E\left(t_{2}\right)\right)}(u, v) \\
& =\int \mathbb{E}\left[\exp \left(i\left\langle\xi_{1}, A(v-u)\right\rangle\right)\right] d P_{\left(E\left(t_{1}\right), E\left(t_{2}\right)\right)}(u, v) \\
& =\int \exp \left((v-u) \psi\left(\xi_{1}\right)\right) d P_{\left(E\left(t_{1}\right), E\left(t_{2}\right)\right)}(u, v), \\
\mathbb{E}\left[\exp \left(i\left\langle\xi_{2}, M\left(t_{1}\right)\right\rangle\right)\right] & =\int \mathbb{E}\left[\exp \left(i\left\langle\xi_{2}, A(u)\right\rangle\right)\right] d P_{E\left(t_{1}\right)}(u) \\
& =\int \exp \left(u \psi\left(\xi_{2}\right)\right) d P_{E\left(t_{1}\right)}(u) .
\end{aligned}
$$

Hence

$$
\tilde{R}\left(t_{1}, t_{2} ; \xi_{1}, \xi_{2}\right)=\int \exp \left((v-u) \psi\left(\xi_{1}\right)\right) d P_{\left(E\left(t_{1}\right), E\left(t_{2}\right)\right)}(u, v) \cdot \int \exp \left(u \psi\left(\xi_{2}\right)\right) d P_{E\left(t_{1}\right)}(u) .
$$

Now fix any $\tilde{\xi}_{1}, \tilde{\xi}_{2} \in \mathbb{R}^{d} \backslash\{0\}$ and let $\xi_{1}=s_{1}^{E^{*}} \tilde{\xi}_{1}$ and $\xi_{2}=s_{2}^{E^{*}} \tilde{\xi}_{2}$ for $s_{1}, s_{2}>0$. Note that since $\nu$ is full and operator-stable, we have $|\hat{\nu}(\xi)|=|\exp (\psi(\xi))|=\exp (\operatorname{Re} \psi(\xi))<1$ (see, e.g., $[22$, Corollary 7.1.12]). Hence $\operatorname{Re} \psi(\xi)<0$ for any $\xi \neq 0$. Using (4.1), we get

$$
\begin{aligned}
& R\left(t_{1}, t_{2} ; s_{1}, s_{2}\right)=\tilde{R}\left(t_{1}, t_{2} ; s_{1}^{E^{*}} \tilde{\xi}_{1}, s_{2}^{E^{*}} \tilde{\xi}_{2}\right) \\
& \quad=\int \exp \left((v-u) s_{1} \psi\left(\tilde{\xi}_{1}\right)\right) d P_{\left(E\left(t_{1}\right), E\left(t_{2}\right)\right)}(u, v) \cdot \int \exp \left(u s_{2} \psi\left(\tilde{\xi}_{2}\right)\right) d P_{E\left(t_{1}\right)}(u) .
\end{aligned}
$$

Note that this expression extends continuously to $s_{1}=0, s_{2}=0$ by dominated convergence. Using dominated convergence together with $\operatorname{Re} \psi(\xi)<0$ again, we get by [21, Corollary 3.2] that for some $C_{\beta}>0$,

$$
\begin{aligned}
F\left(t_{1}, t_{2}\right) & =\left.\frac{\partial^{2}}{\partial s_{2} \partial s_{1}} R\left(t_{1}, t_{2} ; s_{1}, s_{2}\right)\right|_{s_{1}=0, s_{2}=0} \\
& =\psi\left(\tilde{\xi}_{1}\right) \int(v-u) d P_{\left(E\left(t_{1}\right), E\left(t_{2}\right)\right)}(u, v) \cdot \psi\left(\tilde{\xi}_{2}\right) \int u d P_{E\left(t_{1}\right)}(u) \\
& =\psi\left(\tilde{\xi}_{1}\right) \psi\left(\tilde{\xi}_{2}\right) \mathbb{E}\left[E\left(t_{2}\right)-E\left(t_{1}\right)\right] \cdot \mathbb{E}\left[E\left(t_{1}\right)\right] \\
& =C_{\beta}^{2} \psi\left(\tilde{\xi}_{1}\right) \psi\left(\tilde{\xi}_{2}\right)\left(t_{2}^{\beta}-t_{1}^{\beta}\right) t_{1}^{\beta} .
\end{aligned}
$$

On the other hand, using that $\{A(t)\}_{t \geq 0}$ has s.i.i., we get

$$
\begin{aligned}
\tilde{L}\left(t_{1}, t_{2} ; \xi_{1}, \xi_{2}\right) & =\mathbb{E}\left[\exp \left(i\left\langle\xi_{1}, M\left(t_{2}\right)-M\left(t_{1}\right)\right\rangle+i\left\langle\xi_{2}, M\left(t_{1}\right)\right\rangle\right)\right] \\
& =\int \mathbb{E}\left[\exp \left(i\left\langle\xi_{1}, A(v)-A(u)\right\rangle+i\left\langle\xi_{2}, A(u)\right\rangle\right)\right] d P_{\left(E\left(t_{1}\right), E\left(t_{2}\right)\right)}(u, v) \\
& =\int \mathbb{E}\left[\exp \left(i\left\langle\xi_{1}, A(v-u)\right\rangle\right)\right] \cdot \mathbb{E}\left[\exp \left(i\left\langle\xi_{2}, A(u)\right\rangle\right)\right] d P_{\left(E\left(t_{1}\right), E\left(t_{2}\right)\right)}(u, v) \\
& =\int \exp \left((v-u) \psi\left(\xi_{1}\right)\right) \cdot \exp \left(u \psi\left(\xi_{2}\right)\right) d P_{\left(E\left(t_{1}\right), E\left(t_{2}\right)\right)}(u, v) .
\end{aligned}
$$


Let $\xi_{1}=s_{1}^{E^{*}} \tilde{\xi}_{1}$ and $\xi_{2}=s_{2}^{E^{*}} \tilde{\xi}_{2}$ as above to get

$$
\begin{aligned}
L\left(t_{1}, t_{2} ; s_{1}, s_{2}\right) & =\tilde{L}\left(t_{1}, t_{2} ; s_{1}^{E^{*}} \tilde{\xi}_{1}, s_{2}^{E^{*}} \tilde{\xi}_{2}\right) \\
& =\int \exp \left((v-u) s_{1} \psi\left(\tilde{\xi}_{1}\right)\right) \cdot \exp \left(u s_{2} \psi\left(\tilde{\xi}_{2}\right)\right) d P_{\left(E\left(t_{1}\right), E\left(t_{2}\right)\right)}(u, v) .
\end{aligned}
$$

Note that $R\left(t_{1}, t_{2} ; s_{1}, s_{2}\right)=L\left(t_{1}, t_{2} ; s_{1}, s_{2}\right)$. Now, as before,

$$
\begin{aligned}
G\left(t_{1}, t_{2}\right) & =\left.\frac{\partial^{2}}{\partial s_{2} \partial s_{1}} L\left(t_{1}, t_{2} ; s_{1}, s_{2}\right)\right|_{s_{1}=0, s_{2}=0} \\
& =\psi\left(\tilde{\xi}_{1}\right) \psi\left(\tilde{\xi}_{2}\right) \int(v-u) u d P_{\left(E\left(t_{1}\right), E\left(t_{2}\right)\right)}(u, v) \\
& =\psi\left(\tilde{\xi}_{1}\right) \psi\left(\tilde{\xi}_{2}\right)\left(\mathbb{E}\left[E\left(t_{1}\right) E\left(t_{2}\right)\right]-\mathbb{E}\left[E\left(t_{1}\right)^{2}\right]\right) .
\end{aligned}
$$

Since $F\left(t_{1}, t_{2}\right)=G\left(t_{1}, t_{2}\right)$ for all $0<t_{1}<t_{2}$, we also have

$$
\frac{\partial^{2}}{\partial t_{1} \partial t_{2}} F\left(t_{1}, t_{2}\right)=\frac{\partial^{2}}{\partial t_{1} \partial t_{2}} G\left(t_{1}, t_{2}\right)
$$

But by $[21,(3.4)]$ (see also $[6$, Proposition $1(\mathrm{a})]$ ), we have for some constant $C>0$,

$$
\begin{aligned}
\frac{\partial^{2}}{\partial t_{1} \partial t_{2}} G\left(t_{1}, t_{2}\right) & =\psi\left(\tilde{\xi}_{1}\right) \psi\left(\tilde{\xi}_{2}\right) \frac{\partial^{2}}{\partial t_{1} \partial t_{2}} \mathbb{E}\left[E\left(t_{1}\right) E\left(t_{2}\right)\right] \\
& =C \psi\left(\tilde{\xi}_{1}\right) \psi\left(\tilde{\xi}_{2}\right)\left(t_{1} t_{2}\right)^{\beta-1}\left(1-\frac{t_{1}}{t_{2}}\right)^{\beta-1}, \\
\frac{\partial^{2}}{\partial t_{1} \partial t_{2}} F\left(t_{1}, t_{2}\right) & =C_{\beta}^{2} \beta^{2} \psi\left(\tilde{\xi}_{1}\right) \psi\left(\tilde{\xi}_{2}\right)\left(t_{1} t_{2}\right)^{\beta-1} .
\end{aligned}
$$

Since $\psi(\xi) \neq 0$ for $\xi \neq 0$, this gives a contradiction.

The limiting process $\{M(t)\}_{t \geq 0}$ of a single-particle CTRW has the following properties.

(S1) Operator self-similarity:

$$
\{M(c t)\}_{t \geq 0} \stackrel{f . d .}{=}\left\{c^{F} M(t)\right\}_{t \geq 0} \text { for any } c>0,
$$

where $F=\beta E$.

(S2) The distribution of $M(t)$ is not operator-stable (see [21, Theorem 4.6]) and hence $\{M(t)\}_{t \geq 0}$ is not an operator-stable process.

(S3) The process $\{M(t)\}_{t \geq 0}$ has neither stationary (see [21, Corollary 4.5]) nor independent increments (see Theorem 4.1).

(S4) Let $h(x, t)$ denote the density of $M(t)$. Then $h$ is the solution to the fractional diffusion equation

$$
\frac{\partial^{\beta}}{\partial t^{\beta}} h(x, t)=L_{x} h(x, t)+\delta(x) \frac{t^{-\beta}}{\Gamma(1-\beta)}, \quad h(x, 0)=\delta(x),
$$


where $L_{x}$ denotes the generator of the Lévy motion $\{A(t)\}_{t \geq 0}$ and the fractional derivative of order $\beta$ is given by

$$
\frac{\partial^{\beta}}{\partial t^{\beta}} h(x, t)=C \int_{0}^{t} \frac{\partial}{\partial t} h(x, \tau)(t-\tau)^{-\beta} d \tau
$$

See $[1,21]$ for details and [26] for a comprehensive treatment of fractional calculus. By [21, Corollary 4.4 and Theorem 5.1], the solution of (4.13) is given by

$$
h(x, t)=\int_{0}^{\infty} p\left(x,(t / s)^{\beta}\right) g_{\beta}(s) d s,
$$

where $p(x, t)$ is the density of $A(t)$ and $g_{\beta}$ is the density of the $\beta$-stable random variable $D(1)$.

Interpretation of the first model. Due to the nonlocal nature of the fractional time derivative in (4.13), a possible interpretation is that the moving particle has a memory. This is also reflected by the fact that $M(t)=A(E(t))$ and $E(t)$ is a stopping time for the $\beta$ stable subordinator, so $\{E(t) \leq s\}$ is measurable with respect to the $\sigma$-field generated by $\{D(u): 0 \leq u \leq s\}$.

For comparison, we now collect in the same order some of the properties of the multiple-particle limiting process $\{\tilde{M}(t)\}_{t \geq 0}$ obtained in this paper. Recall from Section 3 that $\tilde{M}(t)=A\left(C t^{\beta}\right)$ for some $C>0$ and an operator Lévy motion $\{A(t)\}_{t \geq 0}$ modelling the jumps. Then we have the following properties.

(M1) Operator self-similarity:

$$
\{\tilde{M}(c t)\}_{t \geq 0} \stackrel{f . d .}{=}\left\{c^{F} \tilde{M}(t)\right\}_{t \geq 0} \quad \text { for any } c>0,
$$

where $F=\beta E$.

(M2) The distribution of $\tilde{M}(t)$ is operator-stable and $\{\tilde{M}(t)\}_{t \geq 0}$ is an operator-stable process.

(M3) The process $\{\tilde{M}(t)\}_{t \geq 0}$ has independent but nonstationary increments with

$$
\tilde{M}(t)-\tilde{M}(s) \stackrel{d}{=} C\left(t^{\beta}-s^{\beta}\right)^{E} A(1)
$$

for $0<s<t$.

(M4) Let $q(x, t)$ denote the density of $\tilde{M}(t)$. Then it is shown in (3.37) that $q(x, t)$ solves the equation

$$
\frac{\partial}{\partial t} q(x, t)=L_{x}(t) q(x, t), \quad q(x, 0)=\delta(x)
$$

where $L_{x}(t)=C t^{\beta-1} L_{x}$ and $L_{x}$ is the generator of $\{A(t)\}_{t \geq 0}$. Note that by Remark 3.7,

$$
q(x, t)=p\left(x, C_{\beta} t^{\beta}\right)
$$

where $p(x, t)$ is the density of $A(t)$. 
Interpretation of the second model. The cloud of particles is carrying a nonstandard watch running at $t^{\beta}$ ( $t$ is standard time), so the physical time for the particles is deformed. This is reflected by the time-dependent pseudodifferential operator $L_{x}(t)$ for the space variables and even more by $\tilde{M}(t)=A\left(C t^{\beta}\right)$.

In summary, both limiting processes $\{M(t)\}_{t \geq 0}$ and $\{\tilde{M}(t)\}_{t \geq 0}$ are operator self-similar with the same exponent $F=\beta E$, so they model anomalous diffusions since $0<\beta<1$. However, the distributional and process properties of $\{\tilde{M}(t)\}_{t \geq 0}$ are much nicer than those of the single-particle limiting process $\{M(t)\}_{t \geq 0}$. The multiple-particle model suggests that the cloud of particles is moving along the deformed time $t^{\beta}$, whereas the singleparticle model suggests that the particle has a memory.

Remark 4.2. In order to compare our results with [32], we consider the special case of $\{A(t)\}_{t \geq 0}$ being a one-dimensional symmetric $\alpha$-stable Lévy motion. Since $A(t) \stackrel{d}{=}$ $t^{1 / \alpha} A(1)$, it follows easily that for the density $p(x, t)$ of $A(t)$, we have $p(x, t)=t^{-1 / \alpha} p\left(t^{-1 / \alpha} x\right.$, $1)$. Hence, by (4.15) for some constant $C>0$,

$$
h(0, t) \sim C t^{-\beta / \alpha} \quad \text { as } t \rightarrow \infty
$$

if $\alpha>1$, which coincides with [32, (49)]. Moreover, in [32], using a different method, the asymptotic behavior $h(0, t) \sim C t^{-\beta}$ in the case $0<\alpha<1$ is also obtained. It is interesting to note that for the density $q(x, t)$ of our multiple-particle limit process $\tilde{M}(t)$, we have by (4.19) that $q(0, t) \sim C t^{-\beta / \alpha}$ as $t \rightarrow \infty$ independent of $0<\alpha<2$.

\section{Example of a coupled multiple-particle CTRW}

Complementary to the so-called uncoupled CTRWs, where the waiting time $J_{i}$ is assumed to be independent from the ith jump $Y_{i}$, considered in [21] and analyzed further in the present paper, there are also investigations in the so-called coupled case, where dependence between $J_{i}$ and $Y_{i}$ is allowed. In [5], we derived a limit theorem for coupled CTRWs allowing arbitrary dependence between the waiting time $J_{i}$ and the jump $Y_{i}$, by assuming that $\left(Y_{1}, J_{1}\right)$ belongs to some generalized domain of attraction. It is natural to investigate multiple-particle coupled CTRWs similar to the uncoupled case considered in this work. Of course, the introduction of dependence into the model complicates the analysis as can be seen in [5]. We show now, by computing a specific example, that it is possible to get a limit theorem for the coupled case at least for one fixed time point $t>0$ as well. Our example is based on [5, Example 5.6] and we use the notation used there.

Let $D$ be $\beta$-stable for some $0<\beta<1$ and let $A$ be a symmetric, that is, $A \stackrel{d}{=}-A$, operator-stable random vector with exponent $E$. Let $\{A(t)\}_{t \geq 0}$ be the operator Lévy motion generated by $A$. We define the distribution of the jumps $Y$ by the conditional distribution $(Y \mid D=t) \stackrel{d}{=} A(t)$. Now let $\left(Y_{i}^{(k)}, J_{i}^{(k)}\right), i, k \geq 1$, be i.i.d. as $(Y, D)$. Define $X^{(k)}(t)=$ $S^{(k)}\left(N_{t}^{(k)}\right)$ as in Section 2 and note that in contrast to the uncoupled case considered in this paper, now $\left\{S^{(k)}(t)\right\}_{t \geq 0}$ and $\left\{N_{t}^{(k)}\right\}_{t \geq 0}$ are dependent for any fixed $k \geq 1$ but $\left\{X^{(k)}(t)\right\}_{t \geq 0}$ are i.i.d. It is shown in [5, Example 5.6] that for any fixed $t>0$, we have

$$
c^{-E} X^{(k)}(c t) \Rightarrow M^{(k)}(t) \quad \text { as } c \longrightarrow \infty
$$


for all $k \geq 1$. Moreover, $M^{(k)}(t) \stackrel{d}{=} A^{(k)}\left(t B^{(k)}\right)$, where $\left\{A^{(k)}(t)\right\}_{t \geq 0}$ are i.i.d. as $\{A(t)\}_{t \geq 0}$ and $B^{(k)}$ are i.i.d. having a $\beta$-distribution with probability density $u^{\beta-1}(1-u)^{-\beta} /(\Gamma(\beta) \Gamma(1-$ $\beta)$ ) on $(0,1)$ and $\left\{A^{(k)}(t)\right\}_{t \geq 0}, B^{(k)}, k \geq 1$, are independent. Hence

$$
c^{-E} \sum_{k=1}^{n} X^{(k)}(c t) \Longrightarrow \sum_{k=1}^{n} A^{(k)}\left(t B^{(k)}\right) \quad \text { as } c \longrightarrow \infty .
$$

By a variant of (3.7) and $A(c t) \stackrel{d}{=} c^{E} A(t)$, we have

$$
n^{-E} \sum_{k=1}^{n} A^{(k)}\left(t B^{(k)}\right) \stackrel{d}{=} n^{-E} A\left(t \sum_{k=1}^{n} B^{(k)}\right) \stackrel{d}{=} A\left(t \frac{1}{n} \sum_{k=1}^{n} B^{(k)}\right) .
$$

Since by the weak law of large numbers $n^{-1} \sum_{k=1}^{n} B^{(k)} \rightarrow \mathbb{E}\left(B^{(1)}\right)=\beta$ as $n \rightarrow \infty$ in probability, we have shown that for any fixed $t>0$

$$
\mathscr{L}-\lim _{n \rightarrow \infty} \lim _{c \rightarrow \infty}(c n)^{-E} n Z_{n}(c t)=A(\beta t)
$$

The example shows that in the coupled case, the limiting behavior of $Z_{n}(t)$ is quite different from the uncoupled case considered in Section 3. This parallels the differences between the limiting behavior of single-particle uncoupled and coupled CTRWs, respectively. See $[5,21]$. The derivation of a general limit theorem for multiple-particle coupled CTRWs is an open and challenging problem and will be pursued elsewhere.

\section{Acknowledgments}

The authors would like to express thanks to their colleagues, Mark M. Meerschaert, David A. Benson, and Boris Baeumer, from Fractional Calculus Project for pointing out an error in an earlier version of this paper and for some valuable suggestions leading to Remark 3.7. We also thank Prof. Rudolf Gorenflo for a remark leading to the interpretation of the model and thank an anonymous referee for pointing out [32] leading to Remark 4.2.

\section{References}

[1] B. Baeumer and M. M. Meerschaert, Stochastic solutions for fractional Cauchy problems, Fract. Calc. Appl. Anal. 4 (2001), no. 4, 481-500.

[2] E. Barkai, R. Metzler, and J. Klafter, From continuous time random walks to the fractional FokkerPlanck equation, Phys. Rev. E (3) 61 (2000), no. 1, 132-138.

[3] P. Becker-Kern, M. M. Meerschaert, and H.-P. Scheffler, Hausdorff dimension of operator stable sample paths, Monatsh. Math. 140 (2003), no. 2, 91-101.

[4] Limit theorem for continuous-time random walks with two time scales, J. Appl. Probab. 41 (2004), no. 2, 455-466.

[5] Limit theorems for coupled continuous time random walks, Ann. Probab. 32 (2004), no. 1B, 730-756.

[6] N. H. Bingham, Limit theorems for occupation times of Markov Processes, Z. Wahrscheinlichkeitstheorie und Verw. Gebiete 17 (1971), 1-22. 
[7] L. R. G. Fontes, M. Isopi, and C. M. Newman, Random walks with strongly inhomogeneous rates and singular diffusions: convergence, localization and aging in one dimension, Ann. Probab. 30 (2002), no. 2, 579-604.

[8] R. Hilfer, Exact solutions for a class of fractal time random walks, Fractals 3 (1995), no. 1, 211216.

[9] R. Hilfer (ed.), Applications of Fractional Calculus in Physics, World Scientific Publishing, New Jersey, 2000.

[10] R. Hilfer and L. Anton, Fractional master equations and fractal time random walks, Phys. Rev. E (3) 51 (1995), R848-R851.

[11] N. Jacob, Pseudo-Differential Operators and Markov Processes, Mathematical Research, vol. 94, Akademie Verlag, Berlin, 1996.

[12] K. Kawazu and H. Kesten, On birth and death processes in symmetric random environment, J. Statist. Phys. 37 (1984), no. 5-6, 561-576.

[13] J. Klafter, A. Blumen, and M. F. Shlesinger, Stochastic pathway to anomalous diffusion, Phys. Rev. A (3) 35 (1987), no. 7, 3081-3085.

[14] V. Kolokoltsov, V. Korolev, and V. Uchaikin, Fractional stable distributions, J. Math. Sci. (New York) 105 (2001), no. 6, 2569-2576.

[15] M. Kotulski, Asymptotic distributions of the continuous-time random walks: A probabilistic approach, J. Statist. Phys. 81 (1995), 777-792.

[16] T. J. Kozubowski, M. M. Meerschaert, and H.-P. Scheffler, The operator v-stable laws, Publ. Math. Debrecen 63 (2003), no. 4, 569-585.

[17] M. Maejima, Operator-stable processes and operator fractional stable motions, Probab. Math. Statist. 15 (1995), 449-460.

[18] M. Maejima and J. D. Mason, Operator-self-similar stable processes, Stochastic Process. Appl. 54 (1994), no. 1, 139-163.

[19] M. M. Meerschaert, M. D. Benson, and B. Baeumer, Multidimensional advection and fractional dispersion, Phys. Rev. E (3) 59 (1999), 5026-5028.

[20] - Operator Lévy motion and multiscaling anomalous diffusion, Phys. Rev. E (3) 63 (2001), 021112-021117.

[21] M. M. Meerschaert and H.-P. Scheffler, Limit theorems for continuous time random walks with infinite mean waiting times, to appear in J. Applied Probab.

[22] _ Limit Distributions for Sums of Independent Random Vectors: Heavy Trails in Theory and Practice, Wiley Series in Probability and Statistics, John Wiley \& Sons, New York, 2001.

[23] R. Metzler and J. Klafter, The random walk's guide to anomalous diffusion: a fractional dynamics approach, Phys. Rep. 339 (2000), no. 1, 1-77.

[24] E. W. Montroll and G. H. Weiss, Random walks on lattices. II, J. Math. Phys. 6 (1965), 167-181.

[25] Th. M. Nieuwenhuizen and M. H. Ernst, Excess noise in a hopping model for a resistor with quenched disorder, J. Statist. Phys. 41 (1985), no. 5-6, 773-801.

[26] S. G. Samko, A. A. Kilbas, and O. I. Marichev, Fractional Integrals and Derivatives. Theory and Applications, Gordon and Breach Science Publishers, Yverdon, 1993.

[27] G. Samorodnitsky and M. S. Taqqu, Stable Non-Gaussian Random Processes: Stochastic Models with Infinite Variance, Stochastic Modeling, Chapman \& Hall, New York, 1994.

[28] K. I. Sato, Lévy Processes and Infinitely Divisible Distributions, Cambridge Studies in Advanced Mathematics, vol. 68, Cambridge University Press, Cambridge, 1999.

[29] H. Scher and M. Lax, Stochastic transport in a disordered solid. I. Theory, Phys. Rev. B (3) 7 (1973), no. 10, 4491-4502.

[30] M. F. Shlesinger, J. Klafter, and Y. M. Wong, Random walks with infinite spatial and temporal moments, J. Statist. Phys. 27 (1982), no. 3, 499-512.

[31] V. V. Uchaikin and V. M. Zolotarev, Chance and Stability. Stable Distributions and Their Applications, Modern Probability and Statistics, VSP, Utrecht, 1999. 
[32] H. Weissman, G. H. Weiss, and S. Havlin, Transport properties of the continuous-time random walk with a long-tailed waiting-time density, J. Statist. Phys. 57 (1989), no. 1-2, 301-317.

[33] W. Whitt, Stochastic-Process Limits. An Introduction to Stochastic-Process Limits and Their Application to Queues, Springer Series in Operations Research, Springer-Verlag, New York, 2002.

[34] G. M. Zaslavsky, Fractional kinetic equation for Hamiltonian chaos. Chaotic advection, tracer dynamics and turbulent dispersion, Phys. D 76 (1994), no. 1-3, 110-122.

Peter Becker-Kern: Fachbereich Mathematik, University of Dortmund, 44221 Dortmund, Germany

E-mail address: peter.becker-kern@math.uni-dortmund.de

Hans-Peter Scheffler: Fachbereich Mathematik, University of Dortmund, 44221 Dortmund, Germany

E-mail address: hps@math.uni-dortmund.de 


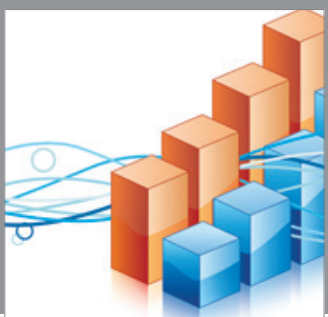

Advances in

Operations Research

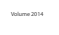

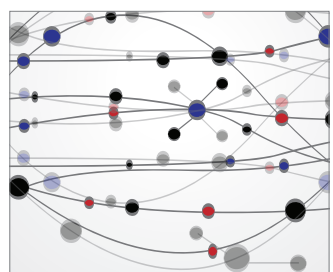

\section{The Scientific} World Journal
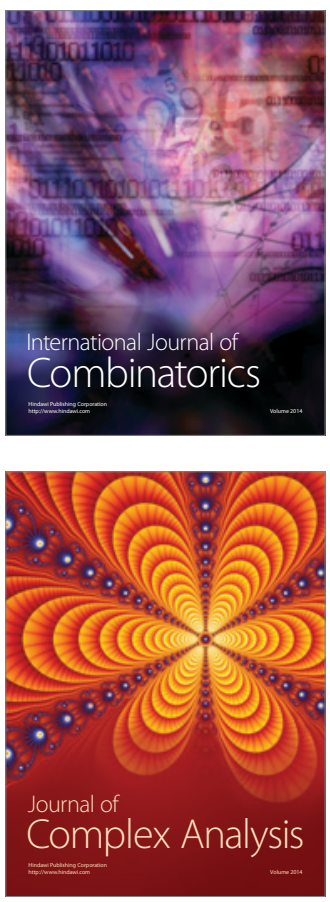

International Journal of

Mathematics and

Mathematical

Sciences
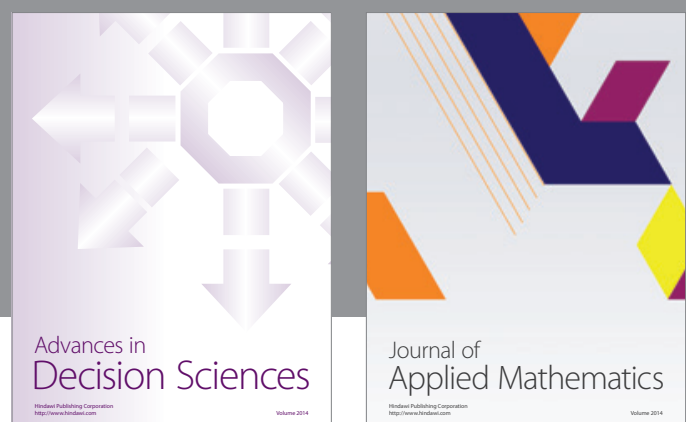

Journal of

Applied Mathematics
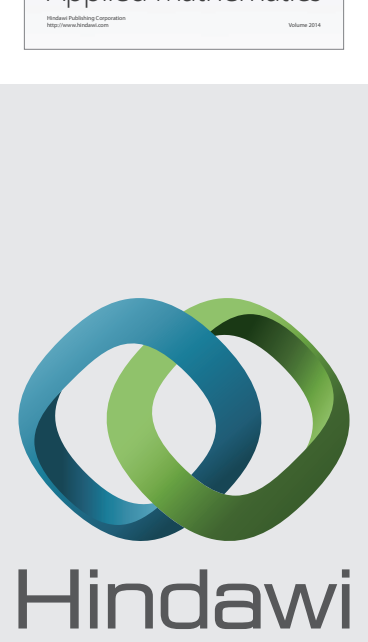

Submit your manuscripts at http://www.hindawi.com
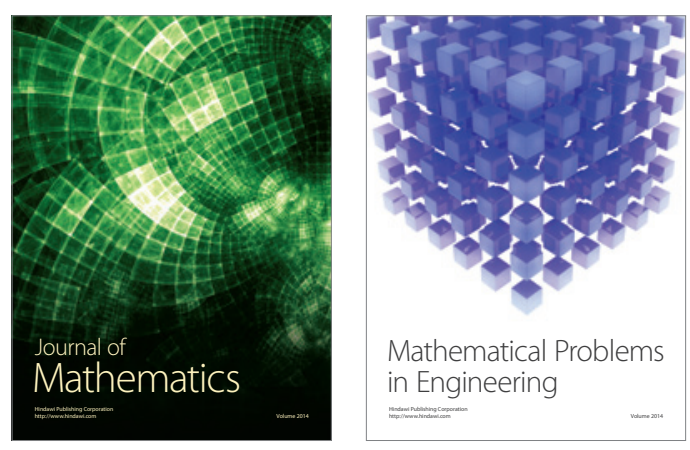

Mathematical Problems in Engineering
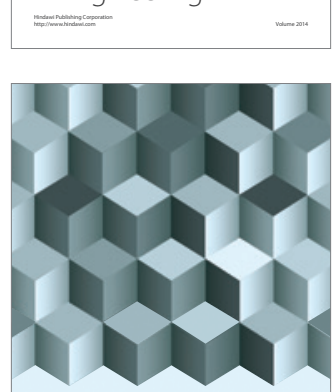

Journal of

Function Spaces
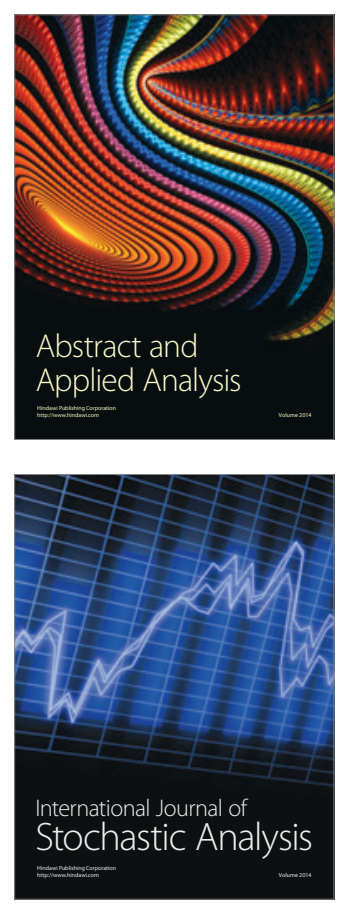

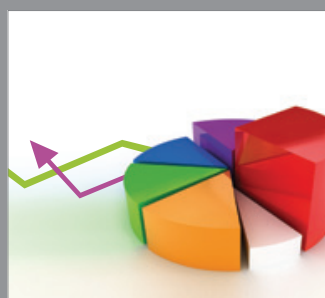

ournal of

Probability and Statistics

Promensencen
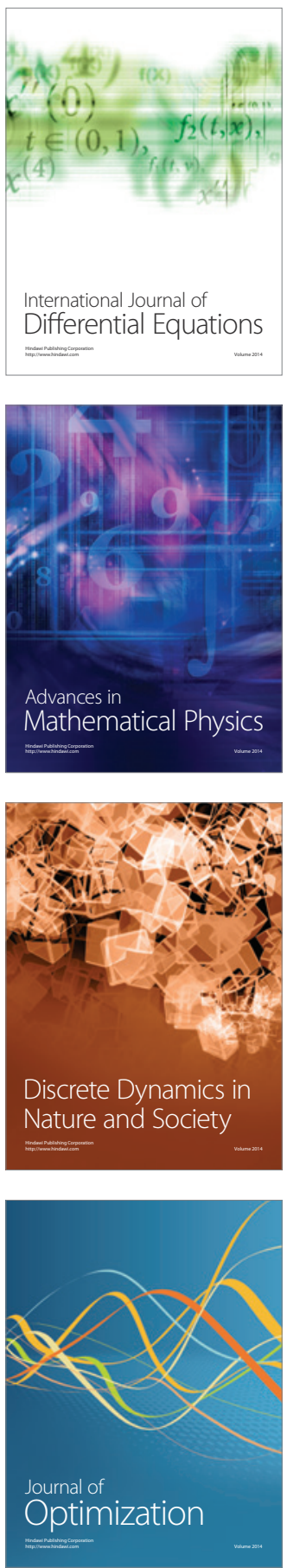